\title{
Situación actual de la formación en archivística en Colombia
}

\author{
Carlos Alberto Zapata Cárdenas *
}

Artículo recibido:

20 de octubre de 2007.

Artículo aceptado:

18 de junio de 2008.

\section{RESUMEN}

La información que se presenta en este artículo recoge los elementos más importantes de la formación archivística en Colombia, tanto a nivel formal como informal. Los resultados que aquí se presentan constituyen una fuente de información valiosa tanto para las instituciones de educación superior que ofrecen formación en Archivística como para otros organismos que ofrecen formación en los diferentes niveles de educación en nuestro país (SENA, Archivo General de la Nación, Biblioteca Nacional, entre otros).

En cuanto a los resultados, el estudio arrojó diferencias marcadas en el desarrollo de la educación en el sector de archivos, así como una alta concentración de la formación en las principales ciudades (Bogotá, Medellín, Ibagué, Tunja, Cali, Bucaramanga, Cartagena, Barranquilla), particularmente en ciudades cercanas

* Universidad de La Salle, Colombia. Czapatca@banrep.gov.co

INVESTIGACIÓN BIBLIOTECOLÓGICA, Vol. 22, Núm. 46, septiembre/diciembre, 2008, México, ISSN: 0187-358X. pp. 139-164 
a los centros de formación universitaria en las que se ofrecen estudios en archivística (Bogotá, Medellín y Armenia), tanto en pregrado como en postgrado. De igual forma se muestra la estratificación en la oferta formativa en la medida en que la demanda se sitúe en poblaciones distantes de dichos centros. En algunas regiones, la única oferta es de tipo no formal y en muchos casos es impartida por instituciones públicas.

Los resultados demuestran que la oferta en el sector de archivos es amplia, lo cual se explica por las políticas públicas que en el campo de los archivos ha formulado el Archivo General de la Nación, y por la sensibilidad que despierta el manejo de la información documental en las organizaciones públicas y privadas.

Finalmente, a través de la investigación de caracterización ocupacional se identificaron elementos de utilidad al definir las competencias laborales del personal de archivos, que a su vez fundamentan el análisis de la evolución del conocimiento archivístico y la comprensión del desarrollo educativo asociado a estas disciplinas, así como en la demanda de recurso calificado este campo profesional y ocupacional.

Palabras Claves: Formación en archivística; Caracterización ocupacional en archivos; formación del archivista; Competencias laborales.

\section{ABSTRACT}

Present situation of Archivist Education in Colombia Carlos Alberto Zapata Cárdenas

This research on the archive's sector in Colombia allows the comprehension of real needs in public and private archives, and in all fields of information sciences, as well as the needs of the market and the educational trends in this field. It also shows differences between educational level in big an medium size cities, where there is a concentration of formal education in the archive field, whereas non formal education predominates in small cities. In addition, the study found several differences in the training and education received by those working in the archives.

Keywords: Archive education; Archivist profile; Work competences. 


\section{INTRODUCCION}

T os resultados de este artículo corresponden a uno de los componentes Lanalizados en una investigación sobre caracterización ocupacional adelantada por la Facultad de Sistemas de Información y Documentación de la Universidad de la Salle, entre los años 2005 y 2007, cuyo objetivo central fue recoger información que permita determinar las características, necesidades y tendencias del sector de archivos en Colombia a partir del análisis de diferentes entornos: organizacional, legal, tecnológico, educativo y ocupacional.

Los objetivos formulados por el grupo investigador fueron:

- Identificar, describir y analizar las características y las tendencias de la oferta formativa en el sector de archivos en Colombia, que sirvan como insumo para la planificación de programas y planes educativos en este sector en los próximos años,

- Evaluar la evolución de la oferta formativa en este sector y su relación con el entorno organizacional, legal y ocupacional.

- Identificar la distribución de la oferta en los diferentes niveles de formación, así como su distribución en todo el país.

- Expresar las tendencias del desarrollo de la educación y capacitación en el sector de archivos en Colombia

\section{Metodología}

\section{Tipo de Investigación}

El estudio realizado es observacional de tipo descriptivo, el cual es definido por Hernández Sampieri (2000) como aquel que “[...] da cuenta de un fenómeno, situación o individuo para dar idea cabal". Los resultados dan cuenta de la situación actual que tienen los archivos desde la perspectiva educativa, y su relación con el entorno ocupacional, tecnológico, legal y organizacional con el sector de archivos del país.

\section{Instrumentos de recolección de información}

Para la recolección de información se aplicó una encuesta estructurada y se realizó una reunión de expertos, lo que permitió recoger la información sobre competencias esperadas, tendencias de la formación, necesidades del sector y perfiles del personal vinculado a los archivos. Para identificar la oferta actual se recurrió a la investigación documental y a la solicitud directa de información 
a las entidades que ofrecen educación formal y no formal en nuestro país, así como a la Internet y a la prensa.

\section{Análisis e interpretación de la información}

El análisis de la información se realizó a través del análisis estadístico y la interpretación de los datos obtenidos. Se correlacionaron los resultados obtenidos y los diferentes entornos en los cuales se dividió el estudio.

\section{ANÁlisis DE LA OFERTA EDUCATIVA EN EL SECTOR DE ARCHIVOS EN COLOMBIA}

\section{Evolución de la oferta formativa}

El primer programa profesional en archivística existente en Colombia se abrió oficialmente en marzo de 1971, en la Universidad de la Salle, y estaba adscrito a la Facultad de Filosofía y Letras (Zapata G, 1977); la siguiente iniciativa fue la creación del Programa de Ciencia de la Información de la Universidad del Quindío, el cual también incluía dentro de su formación asignaturas sobre archivos; este programa se caracterizó por ser inicialmente a distancia (actualmente se imparte en la modalidad virtual).

A nivel tecnológico la Universidad Católica de Manizales y la Universidad de Antioquia ofrecen formación a nivel tecnológico en el campo archivístico. La Fundación Universitaria INPAHU ofrece asignaturas del sector de archivos en su programa de Administración de Sistemas de Información y Documentación, pero no otorga este título. A nivel de postgrado existen tres programas, uno en la Universidad de la Salle, otro en la Universidad Pedagógica y Tecnológica de Colombia y uno más en la Universidad Javeriana.

El cuadro siguiente muestra las fechas de creación de los diferentes programas en archivística tanto a nivel profesional (pregrado y postgrado) como técnico y tecnológico, así:

\begin{tabular}{|c|l|l|}
\hline Año & \multicolumn{1}{|c|}{ Programa } & \multicolumn{1}{|c|}{ Universidad } \\
\hline 1971 & Facultad de Bibliotecología y Archivística & La Salle \\
\hline 1986 & $\begin{array}{l}\text { Programa de Ciencia de la Información, Biblio- } \\
\text { tecología y Archivística }\end{array}$ & Quindío - Tolima \\
\hline 1994 & $\begin{array}{l}\text { Especialización en Sistemas de Información y } \\
\text { Gerencia de Documentos }\end{array}$ & La Salle \\
\hline 1998 & Especialización en Archivística & Pedagógica y Tecnológica de Tunja \\
\hline 1998 & Especialización en Archivística & Javeriana \\
\hline
\end{tabular}




\begin{tabular}{|c|l|l|}
\hline 2001 & $\begin{array}{l}\text { Tecnología en Administración de Sistemas de } \\
\text { Información y Documentación }\end{array}$ & INPAHU \\
\hline 2000 & Tecnología en Documentación y Archivística & Católica de Manizales \\
\hline 2004 & Tecnología en Archivística & Antioquia \\
\hline
\end{tabular}

A nivel técnico, el Servicio Nacional de Aprendizaje(SENA), ofrece formación en archivos, para técnicos y auxiliares (2.500 personas han egresado de sus programas, según cifras de dicha entidad); en cuanto a formación no formal, el Archivo General de la Nación, a través de la División de Programas Especiales, la Sociedad Colombiana de Archivistas y el Colegio Colombiano de Archivistas ofrecen capacitación en los campos relacionados con la gestión de archivos, en su mayor parte dirigida a funcionarios del sector público que no han accedido a la formación formal ya señalada.

En algunas regiones las cajas de compensación familiar también dan capacitación en algunas áreas de la archivística.

\section{Niveles de la oferta de formación}

\section{Educación formal}

Es aquélla que se imparte en establecimientos educativos aprobados, en una secuencia de ciclos lectivos que están sujetos a pautas curriculares progresivas y que conducen a grados y títulos.

En el nivel de formación superior existen tres programas que otorgan a título profesional; de los cuales uno se imparte en modalidad presencial y dos en la virtual. El programa de la Universidad de la Salle cuenta con una acreditación de alta calidad expedida por el Ministerio de Educación mientras que los otros dos tienen un registro calificado (Universidad del Quindío y Universidad del Tolima). Existen tres programas de postgrado (a nivel de especialización).

En la tabla 1 se indican las características más importantes de dichos programas. 
Tabla 1. Oferta formativa del nivel profesional y especialización - Archivos Públicos

\begin{tabular}{|c|c|c|c|c|c|c|c|c|c|c|c|c|}
\hline \multirow{2}{*}{ 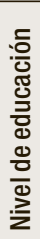 } & \multirow[b]{2}{*}{ Núm } & \multirow[b]{2}{*}{ Nombre del programa } & \multicolumn{3}{|c|}{ Institución } & \multicolumn{3}{|c|}{ Estilo } & \multicolumn{2}{|c|}{ Jornada } & \multirow[b]{2}{*}{ 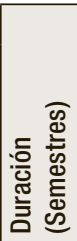 } & \multirow[b]{2}{*}{$\begin{array}{l}\text { Lugar } \\
\text { (sede) }\end{array}$} \\
\hline & & & Nombre & $\frac{\text { 을 }}{\text { 음 }}$ & 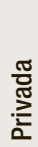 & 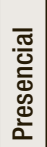 & 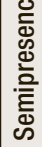 & 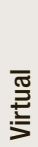 & 을 & 을 & & \\
\hline \multirow{3}{*}{ 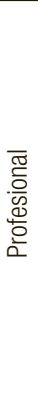 } & 1 & $\begin{array}{l}\text { Sistemas de información } \\
\text { y documentación, biblio- } \\
\text { tecología y archivística }\end{array}$ & La Salle & & 1 & 1 & & & 1 & 1 & 10 & Bogotá \\
\hline & 2 & $\begin{array}{l}\text { Ciencia de la informa- } \\
\text { ción y la documentación, } \\
\text { bibliotecología y archi- } \\
\text { vística }\end{array}$ & Quindío & 1 & & & & 1 & 1 & 1 & 9 & Armenia \\
\hline & 3 & $\begin{array}{l}\text { Ciencia de la informa- } \\
\text { ción y la documentación, } \\
\text { bibliotecología y archi- } \\
\text { vística }\end{array}$ & Tolima & 1 & & & & 1 & 1 & 1 & 9 & Ibagué \\
\hline \multirow{3}{*}{$\begin{array}{l}\frac{0}{ㅇ ㅠ ㅁ ~} \\
\frac{5}{\mathscr{W}} \\
\frac{0}{0}\end{array}$} & 4 & $\begin{array}{l}\text { Especialización en archi- } \\
\text { vística }\end{array}$ & Javeriana & & 1 & 1 & & & & 1 & 2 & Bogotá \\
\hline & 5 & $\begin{array}{l}\text { Especialización en sis- } \\
\text { temas de información y } \\
\text { gerencia de documentos }\end{array}$ & La Salle & & 1 & & 1 & & & 1 & 2 & Bogotá \\
\hline & 6 & $\begin{array}{l}\text { Especialización en archi- } \\
\text { vística }\end{array}$ & UPTC & 1 & & 1 & & & & 1 & 2 & Tunja \\
\hline
\end{tabular}

A nivel técnico y tecnológico la oferta de estudios en archivística está representada en cuatro niveles (Tabla 2), uno técnico con presencia en diferentes regiones del país, y tal vez el de mayor cubrimiento, y tres a nivel tecnológico situados en ciudades con tradición educativa, uno en la Universidad de Antioquia, otro en la Universidad Católica de Manizales y el tercero es la Fundación Universitaria INPAHU, la cual combina la formación es archivos con otras área afines como la Documentación. En promedio, la formación técnica tiene una duración de dos años, mientras que la tecnológica es de tres años (seis semestres académicos). 
Tabla 2. Oferta formativa del nivel técnico y tecnológico - Archivos Públicos

\begin{tabular}{|c|c|c|c|c|c|c|c|c|c|c|c|c|}
\hline \multirow{2}{*}{ 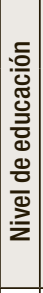 } & \multirow[b]{2}{*}{ Núm. } & \multirow[b]{2}{*}{$\begin{array}{c}\text { Nombre del } \\
\text { programa }\end{array}$} & \multicolumn{3}{|c|}{ Institución } & \multicolumn{3}{|c|}{$\begin{array}{l}\text { Estilo de } \\
\text { educación }\end{array}$} & \multicolumn{2}{|c|}{ Jornada } & \multirow[b]{2}{*}{$\begin{array}{c}\text { Duración } \\
\text { (Semestres) }\end{array}$} & \multirow[b]{2}{*}{$\begin{array}{l}\text { Lugar } \\
\text { (Sede) }\end{array}$} \\
\hline & & & Nombre & 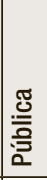 & $\stackrel{\frac{\pi}{2}}{\frac{\pi}{2}}$ & 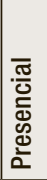 & 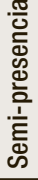 & $\stackrel{\frac{\pi}{5}}{\stackrel{5}{5}}$ & 을 & 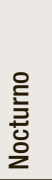 & & \\
\hline 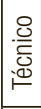 & 1 & $\begin{array}{l}\text { Técnico } \\
\text { profesional en } \\
\text { Archivística }\end{array}$ & SENA & 1 & & 1 & & & 1 & & 2540 Horas & $\begin{array}{l}\text { Sedes } \\
\text { SENA }\end{array}$ \\
\hline \multirow{3}{*}{ 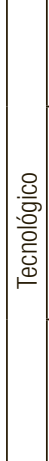 } & 2 & $\begin{array}{l}\text { Tecnología } \\
\text { en } \\
\text { Archivística }\end{array}$ & U. de Antioquia & 1 & & 1 & & & 1 & 1 & 6 Semestres & Medellín \\
\hline & 3 & $\begin{array}{l}\text { Tecnólogo en } \\
\text { Admón. de } \\
\text { Sistemas de } \\
\text { Información } \\
\text { y Documen- } \\
\text { tación }\end{array}$ & INPAHU & & 1 & 1 & & & & 1 & 7 Semestres & Bogotá \\
\hline & 4 & $\begin{array}{l}\text { Tecnología } \\
\text { en Docu- } \\
\text { mentación y } \\
\text { Archivística }\end{array}$ & $\begin{array}{l}\text { Universidad } \\
\text { Católica de } \\
\text { Manizales }\end{array}$ & & 1 & & 1 & & & & 6 semestres & $\begin{array}{l}\text { Maniza- } \\
\text { les }\end{array}$ \\
\hline
\end{tabular}

\section{Educación No formal}

La educación no formal se ofrece con el fin de complementar, actualizar y suplir conocimientos y formar en aspectos académicos o laborales sin sujetarse al sistema de niveles y grados establecidos para la educación formal. En relación con la información recolectada se identificaron 33 programas ofrecidos por diferentes entidades.

Se debe resaltar que en algunos lugares la oferta identificada no es permanente ni sistemática, es decir que tanto la denominación como la duración de los programas depende de las necesidades de la demanda y de los criterios de la entidad oferente. El estudio identifico cuatro tipos de modalidades en la formación: diplomados, cursos, talleres y seminarios. A nivel de diplomados, en la Tabla 3 se aprecia la oferta de capacitación identificada. 
Tabla 3. Oferta de capacitación a través de diplomados - Archivos Públicos

\begin{tabular}{|c|c|c|c|c|c|c|c|c|c|c|c|c|}
\hline \multirow{2}{*}{ 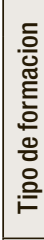 } & \multirow[b]{2}{*}{ Núm. } & \multirow[b]{2}{*}{$\begin{array}{l}\text { Nombre del } \\
\text { programa }\end{array}$} & \multicolumn{3}{|c|}{ Institución } & \multicolumn{3}{|c|}{ Estilo } & \multicolumn{2}{|c|}{ Horario } & \multirow[b]{2}{*}{ Duración } & \multirow[b]{2}{*}{$\begin{array}{l}\text { Lugar } \\
\text { (Sede) }\end{array}$} \\
\hline & & & Nombre & $\frac{\text { 음 }}{\frac{0}{3}}$ & 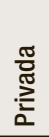 & 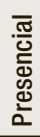 & 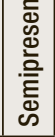 & 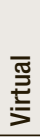 & 을 & 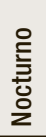 & & \\
\hline \multirow{3}{*}{ 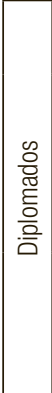 } & 1 & $\begin{array}{l}\text { Gestión de informa- } \\
\text { ción documental }\end{array}$ & \begin{tabular}{|l|} 
U. \\
Javeriana
\end{tabular} & & 1 & 1 & & & & 1 & 120 Horas & Bogotá \\
\hline & 2 & $\begin{array}{l}\text { Gerencia Documen- } \\
\text { tal orientada a la } \\
\text { gestión de archivos } \\
\text { históricos y empre- } \\
\text { sariales }\end{array}$ & $\begin{array}{l}\text { COMFA- } \\
\text { MILIAR- } \\
\text { Atlántico }\end{array}$ & & 1 & 1 & & & 1 & & 90 horas & B/quilla \\
\hline & 3 & $\begin{array}{l}\text { Diplomado en archi- } \\
\text { vística }\end{array}$ & $\begin{array}{l}\text { U. Peda- } \\
\text { gógica y } \\
\text { Tecnológi- } \\
\text { ca de }\end{array}$ & 1 & & 1 & 1 & & & 1 & 150 horas & Tunja \\
\hline
\end{tabular}

Debido a su naturaleza, las asociaciones que agremian a los profesionales y trabajadores del sector de archivos también desarrollan procesos de formación orientados a actualizar a sus asociados, y en general a los interesados y profesionales del sector en áreas propias de su actividad o en las cuales exista una demanda de capacitación. Las Cajas de Compensación Familiar realizan igualmente capacitación en el sector de archivos.

La tabla 4 muestra una descripción de los seminarios que se han realizado en los últimos tres años en el área. En general, los seminarios obedecen al criterio de reiteración; es decir, se trata de áreas que han sido identificadas como de formación continuada y los seminarios se imparten de manera repetitiva o continua (se ofrecen regularmente). Durante los últimos tres años se han ofrecido un total de once (11) seminarios principalmente en áreas como: gestión de documentos, documentos electrónicos, tablas de retención documental, gestión de calidad, auditoria de Información y contratación de servicios.

Los cursos están orientados a desarrollar competencias técnicas y administrativas en el sector; los participantes son principalmente empleados de nivel de base (auxiliares y técnicos), aunque en algunos casos, dependiendo de la temática, también se dirigen a directores o personas de rango intermedio que tienen a su cargo responsabilidades administrativas (dirección y control), en particular si los profesionales no tienen formación disciplinar previa. Los cursos son el tipo de formación que con mayor frecuencia se ofrecen, están dirigidos principalmente a capacitar en la función (saber hacer) y desarrollan un contenido específico siguiendo una secuencia sistemática de una determinada área del conocimiento o de una actividad que se lleva a cabo (Tabla 5). 
Tabla 4. Oferta de capacitación a través de seminarios - Archivos Públicos

\begin{tabular}{|c|c|c|c|c|c|c|c|c|c|c|c|c|}
\hline \multirow{2}{*}{ 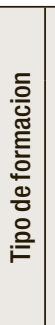 } & \multirow[b]{2}{*}{ Núm. } & \multirow[b]{2}{*}{$\begin{array}{c}\text { Nombre del } \\
\text { programa }\end{array}$} & \multicolumn{3}{|c|}{ Institución } & \multicolumn{3}{|c|}{$\begin{array}{l}\text { Estilo de } \\
\text { educación }\end{array}$} & \multicolumn{2}{|c|}{ Horario } & \multirow[b]{2}{*}{ Duración } & \multirow[b]{2}{*}{$\begin{array}{l}\text { Lugar } \\
\text { (Sede) }\end{array}$} \\
\hline & & & Nombre & 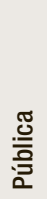 & 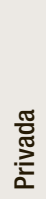 & 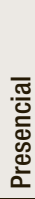 & 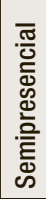 & 矛 & 号 & $\begin{array}{l}\text { 은 } \\
\text { 总 } \\
\text { 을 }\end{array}$ & & \\
\hline \multirow{11}{*}{ 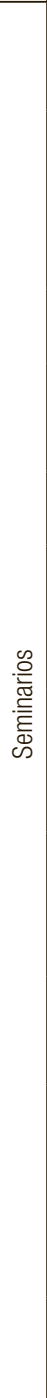 } & 1 & \begin{tabular}{|l|} 
Seminario de \\
Archivos Orales
\end{tabular} & \begin{tabular}{|l|} 
Archivo \\
General de la \\
Nación(AGN)
\end{tabular} & 1 & & 1 & & & 1 & & 24 horas & Bogotá \\
\hline & 2 & $\begin{array}{l}\text { Seminario de } \\
\text { Archivos de } \\
\text { Entidades de } \\
\text { Educación Básica } \\
\text { y Media. }\end{array}$ & AGN & 1 & & 1 & & & 1 & & 24 horas & Bogotá \\
\hline & 3 & $\begin{array}{l}\text { Seminario de Ar- } \\
\text { chivos Notariales }\end{array}$ & AGN & 1 & & 1 & & & 1 & & 24 horas & Bogotá \\
\hline & 4 & $\begin{array}{l}\text { Los Documentos y } \\
\text { Archivos Electró- } \\
\text { nicos" }\end{array}$ & AGN & 1 & & 1 & & & 1 & & 24 horas & Bogotá \\
\hline & 5 & $\begin{array}{l}\text { Seminario de Ar- } \\
\text { chivos Contables }\end{array}$ & AGN & 1 & & 1 & & & 1 & & 24 horas & Bogotá \\
\hline & 6 & $\begin{array}{l}\text { Auditoria de } \\
\text { Información }\end{array}$ & $\begin{array}{l}\text { Sociedad } \\
\text { Colombiana de } \\
\text { Archivistas } \\
\text { (SCA) }\end{array}$ & & 1 & 1 & & & 1 & & 8 horas & Bogotá \\
\hline & 7 & $\begin{array}{l}\text { Planeación de Sis- } \\
\text { temas de Gestión } \\
\text { Electrónica de } \\
\text { Documentos }\end{array}$ & SCA & & 1 & 1 & & & 1 & & 16 horas & Bogotá \\
\hline & 8 & \begin{tabular}{|l|} 
Diseño e imple- \\
mentación de Ta- \\
blas de retención \\
documental (TRD)
\end{tabular} & SCA & & 1 & 1 & & & 1 & & 16 horas & Bogotá \\
\hline & 9 & \begin{tabular}{|l|} 
Diseño de Siste- \\
mas de Gestión \\
Documental
\end{tabular} & SCA & & 1 & 1 & & & 1 & & 16 horas & Bogotá \\
\hline & 10 & $\begin{array}{l}\text { Implementa- } \\
\text { ción de Tablas } \\
\text { de retención } \\
\text { documental (TRD) } \\
\text { en documentos } \\
\text { electrónicos }\end{array}$ & SCA & & 1 & 1 & & & 1 & & 16 horas & Bogotá \\
\hline & 11 & \begin{tabular}{|l} 
Seminario de \\
Contratación en \\
Archivos \\
\end{tabular} & SCA & & 1 & 1 & & & 1 & & 16 horas & Bogotá \\
\hline
\end{tabular}


Tabla 5. Oferta de capacitación a través de cursos - Archivos Públicos

\begin{tabular}{|c|c|c|c|c|c|c|c|c|c|c|c|c|}
\hline \multirow{2}{*}{ 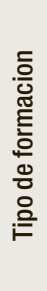 } & \multirow[b]{2}{*}{ Núm. } & \multirow[b]{2}{*}{$\begin{array}{l}\text { Nombre del } \\
\text { programa }\end{array}$} & \multicolumn{3}{|c|}{ Institución } & \multicolumn{3}{|c|}{$\begin{array}{c}\text { Estilo de } \\
\text { educación }\end{array}$} & \multicolumn{2}{|c|}{ Horario } & \multirow[b]{2}{*}{ Duración } & \multirow[b]{2}{*}{$\begin{array}{l}\text { Lugar } \\
\text { (Sede) }\end{array}$} \\
\hline & & & Nombre & 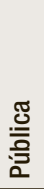 & $\frac{\pi}{\mathbb{T}}$ & 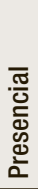 & 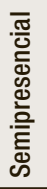 & 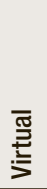 & 을 & $\begin{array}{l}\text { 을 } \\
\text { 总 } \\
\text { 을 }\end{array}$ & & \\
\hline \multirow{13}{*}{ 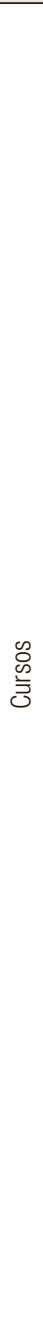 } & 1 & $\begin{array}{l}\text { Fundamentos de } \\
\text { administración } \\
\text { documental }\end{array}$ & SENA & 1 & & & & 1 & 1 & & 60 Horas & $\begin{array}{l}\text { Sedes } \\
\text { SENA }\end{array}$ \\
\hline & 2 & $\begin{array}{l}\text { Auxiliar de oficina } \\
\text { con énfasis en } \\
\text { Documentación y } \\
\text { Archivo }\end{array}$ & $\begin{array}{l}\text { COLSUB- } \\
\text { SIDIO }\end{array}$ & & 1 & 1 & & & & 1 & 320 Horas & Bogotá \\
\hline & 3 & $\begin{array}{l}\text { Administración de } \\
\text { archivo y manejo } \\
\text { de documentos }\end{array}$ & $\begin{array}{l}\text { COMFA- } \\
\text { MILIAR - } \\
\text { Quindío }\end{array}$ & & 1 & 1 & & & & 1 & 24 Horas & Armenia \\
\hline & 4 & $\begin{array}{l}\text { Curso Básico } \\
\text { sobre Organiza- } \\
\text { ción y Manejo de } \\
\text { Archivos. Nivel } \\
1,2 \text { y } 3\end{array}$ & AGN & 1 & & 1 & & & 1 & & 40 horas & Bogotá \\
\hline & 5 & $\begin{array}{l}\text { Técnicas de ofici- } \\
\text { na: Organización } \\
\text { de archivos }\end{array}$ & $\begin{array}{l}\text { COMFA- } \\
\text { MA }\end{array}$ & & 1 & 1 & & & & & 60 horas & Medellín \\
\hline & 6 & $\begin{array}{l}\text { Básico de } \\
\text { Archivística y } \\
\text { Secretariado }\end{array}$ & SENA & 1 & & 1 & & & 1 & & 290 Horas & $\begin{array}{l}\text { Sedes } \\
\text { SENA }\end{array}$ \\
\hline & 7 & $\begin{array}{l}\text { Servicios de } \\
\text { Documentos }\end{array}$ & SENA & 1 & & 1 & & & 1 & & 200 Horas & $\begin{array}{l}\text { Sedes } \\
\text { SENA }\end{array}$ \\
\hline & 8 & $\begin{array}{l}\text { Introducción a la } \\
\text { Archivística }\end{array}$ & SENA & 1 & & 1 & & & 1 & & 90 Horas & $\begin{array}{l}\text { Sedes } \\
\text { SENA }\end{array}$ \\
\hline & 9 & $\begin{array}{l}\text { Procesos } \\
\text { Archivísticos }\end{array}$ & SENA & 1 & & 1 & & & 1 & & 100 Horas & $\begin{array}{l}\text { Sedes } \\
\text { SENA }\end{array}$ \\
\hline & 10 & $\begin{array}{l}\text { Administración de } \\
\text { Archivos }\end{array}$ & SENA & 1 & & 1 & & & 1 & & 400 Horas & $\begin{array}{l}\text { Sedes } \\
\text { SENA }\end{array}$ \\
\hline & 11 & $\begin{array}{l}\text { Sistemas de } \\
\text { Administración de } \\
\text { Documentos }\end{array}$ & SENA & 1 & & 1 & & & 1 & & 380 Horas & $\begin{array}{l}\text { Sedes } \\
\text { SENA }\end{array}$ \\
\hline & 12 & $\begin{array}{l}\text { Organización de } \\
\text { fondos documen- } \\
\text { tales acumulados }\end{array}$ & $\begin{array}{l}\text { Archivo } \\
\text { Distrital }\end{array}$ & 1 & & 1 & & & 1 & & - & Bogotá \\
\hline & 13 & $\begin{array}{l}\text { Aplicación de } \\
\text { TRD }\end{array}$ & $\begin{array}{l}\text { Archivo } \\
\text { Distrital }\end{array}$ & 1 & & 1 & & & 1 & & - & Bogotá \\
\hline
\end{tabular}


En cuanto a los talleres, cuya modalidad busca brindar competencias técnicas para realizar una determinada actividad o proceso a partir de la combinación de teoría y práctica (Tabla 6), la oferta identificada se ubica en su totalidad en la ciudad de Bogotá.

Tabla 6. Oferta de capacitación a través de Talleres - Archivos Públicos

\begin{tabular}{|c|c|c|c|c|c|c|c|c|c|c|c|c|}
\hline \multirow{2}{*}{ 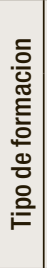 } & \multirow[b]{2}{*}{ Núm. } & \multirow[b]{2}{*}{ Nombre del programa } & \multicolumn{3}{|c|}{ Institución } & \multicolumn{3}{|c|}{$\begin{array}{c}\text { Estilo de } \\
\text { educación }\end{array}$} & \multicolumn{2}{|c|}{ Horario } & \multirow[b]{2}{*}{ Duración } & \multirow[b]{2}{*}{$\begin{array}{l}\text { Lugar } \\
\text { (Sede) }\end{array}$} \\
\hline & & & Nombre & $\frac{\mathbb{J}}{\frac{.}{2}}$ & 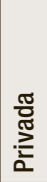 & 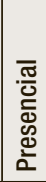 & 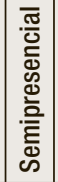 & $\stackrel{\text { 똘 }}{\frac{3}{5}}$ & 号 & $\begin{array}{l}\text { 을 } \\
\text { 壱 } \\
\text { 을 }\end{array}$ & & \\
\hline \multirow{6}{*}{ 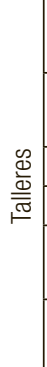 } & 1 & $\begin{array}{l}\text { Organización de Fondos } \\
\text { Acumulados }\end{array}$ & AGN & 1 & & 1 & & & 1 & & 40 horas & Bogotá \\
\hline & 2 & $\begin{array}{l}\text { Taller sobre Conservación } \\
\text { preventiva en archivos }\end{array}$ & AGN & 1 & & 1 & & & 1 & & 40 horas & Bogotá \\
\hline & 3 & Taller de Microfilmación & AGN & 1 & & 1 & & & 1 & & 40 horas & Bogotá \\
\hline & 4 & Taller de Encuadernación & AGN & 1 & & 1 & & & 1 & & 40 horas & Bogotá \\
\hline & 5 & $\begin{array}{l}\text { Seminario Taller de } \\
\text { Archivos }\end{array}$ & U. La Salle & & 1 & 1 & & & & 1 & 40 Horas & Bogotá \\
\hline & 6 & \begin{tabular}{|l|} 
Taller de Auditoría para la \\
Implementación de TRD
\end{tabular} & SCA & & 1 & 1 & & & 1 & & 16 horas & Bogotá \\
\hline
\end{tabular}

\section{Ubicación geográfica de la formación en archivos}

Uno de los resultados más relevantes del estudio tiene que ver con la localización de los programas de formación en sus diferentes modalidades. En la Tabla 7 se puede observar la alta concentración de la oferta educativa en el sector de archivos en la ciudad de Bogotá, algo que está directamente relacionado con la demanda de personal calificado por un lado y por la cantidad de empresas de diferente tipo que tiene sede en la capital.

Aunque el Archivo General de la Nación hace presencia en otras ciudades, esta oferta no puedo ser identificada de manera más precisa, aunque el estudio identificó once (11) tipos de programas que se ofrecen en ciudades diferentes a Bogotá, Medellín y Barranquilla. Se identificaron programas de capacitación en ciudades como Cali, Tunja, y Cartagena, ofrecidos principalmente por universidades o cajas de compensación familiar.

El tipo de formación que más prevalece es el curso, seguido del seminario y los talleres; se observó también que la educación formal tiene menor cobertura que la educación no formal, observándose presencia de educación formal 
en ciudades como Medellín y Tunja que hace algunos años no contaban con oferta en esta área.

Tabla 7. Ubicación de los programas de formación y capacitación

\begin{tabular}{|c|c|c|c|c|c|c|c|c|c|}
\hline \multirow[b]{2}{*}{ Lugar } & \multirow[b]{2}{*}{ Cant. } & \multicolumn{2}{|c|}{ Formal } & \multicolumn{6}{|c|}{ No formal } \\
\hline & & 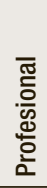 & 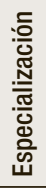 & 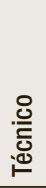 & $\begin{array}{l}\text { 음 } \\
\text { 응 } \\
\text { 덩 } \\
\end{array}$ & 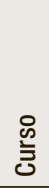 & $\frac{\grave{\varpi}}{\stackrel{\bar{\sigma}}{\leftrightarrows}}$ & $\begin{array}{l}\text { 음 } \\
\text { ㄷ } \\
\text { 음 } \\
\text { ㅁ }\end{array}$ & 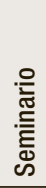 \\
\hline Bogotá & 27 & 1 & 3 & & 1 & 4 & 6 & 1 & 11 \\
\hline Medellín & 2 & & & & & 11 & & & \\
\hline Barranquilla & 1 & & & & & & & 1 & \\
\hline Tunja & 2 & & 1 & & & & & 1 & \\
\hline Nacional $^{1}$ & 11 & 2 & & 1 & & 8 & & & \\
\hline Total & 43 & 3 & 4 & 1 & 2 & 13 & 6 & 2 & 11 \\
\hline
\end{tabular}

\section{Titulaciones ofrecidas en los diferentes programa de formación conducentes a un título}

El desarrollo de las políticas públicas en materia archivística ha propiciado la apertura de programas de formación en el área. En relación con la educación formal, las denominaciones de los programas y los títulos que otorgan varían de una entidad educativa a otra, lo cual obedece a una estrategia de mercadeo que facilita la atracción de nuevos segmentos de candidatos, y en particular a jóvenes recién egresados del bachillerato.

Tabla 8. Denominación de los títulos otorgados

\begin{tabular}{|l|l|l|}
\hline \multicolumn{2}{|c|}{ Denominación de los programas de formación en pregrado } \\
\hline \multicolumn{1}{|c|}{ Universidad } & \multicolumn{1}{|c|}{ Programa } & \multicolumn{1}{c|}{ Título que Otorga } \\
\hline Universidad de la Salle & $\begin{array}{l}\text { Sistemas de información y Docu- } \\
\text { mentación }\end{array}$ & $\begin{array}{l}\text { Profesional en sistemas de Infor- } \\
\text { mación, bibliotecología y archivís- } \\
\text { tica }\end{array}$ \\
\hline Pontificia Universidad Javeriana & $\begin{array}{l}\text { Ciencia de la información - Biblio- } \\
\text { tecología }\end{array}$ & $\begin{array}{l}\text { Profesional en Ciencia de la Infor- } \\
\text { mación - Bibliotecólogo(a). }\end{array}$ \\
\hline Universidad del Quindío & $\begin{array}{l}\text { Ciencia de la Información y la } \\
\text { Documentación, Bibliotecología y } \\
\text { Archivística }\end{array}$ & $\begin{array}{l}\text { Profesional en Ciencia de la Infor- } \\
\text { mación y la Documentación, Bi- } \\
\text { bliotecología y Archivística }\end{array}$ \\
\hline
\end{tabular}

1 El ítem de "Nacional”, relaciona los programas de formación virtual y las instituciones que ofrecen el programa en diferentes sedes distribuidas en el territorio Colombiano, e incluye la formación de instituciones ubicadas en Armenia e Ibagué, por ser esta formación en la modalidad virtual. 


\begin{tabular}{|l|l|l|}
\hline \multicolumn{3}{|c|}{ Denominación de los programas de formación en postgrado } \\
\hline \multicolumn{1}{|c|}{ Universidad } & \multicolumn{1}{|c|}{ Programa } & \multicolumn{1}{c|}{ Título que Otorga } \\
\hline Universidad de la Salle & $\begin{array}{l}\text { Especialización en sistemas de } \\
\text { información y gerencia de docu- } \\
\text { mentos. }\end{array}$ & $\begin{array}{l}\text { Especialista en sistemas de infor- } \\
\text { mación y gerencia de documentos. }\end{array}$ \\
\hline $\begin{array}{l}\text { Universidad Javeriana } \\
\text { Universidad politécnica y tecnoló- } \\
\text { gica de Colombia }\end{array}$ & Especialización en archivística & Especialista en archivística \\
\hline
\end{tabular}

Por su parte, a nivel técnico y tecnológico las titulaciones que se ofrecen son:

Tabla 9. Denominación de los títulos a nivel técnico y tecnológica

\begin{tabular}{|l|l|l|}
\hline \multicolumn{3}{|c|}{ Denominación de los programas de formación tecnológica } \\
\hline \multicolumn{1}{|c|}{ Universidad } & \multicolumn{1}{|c|}{ Programa } & \multicolumn{1}{c|}{ Título que Otorga } \\
\hline Universidad de Antioquia & Tecnología en Archivística & Tecnólogo en Archivística \\
\hline Universidad Católica de Manizales & $\begin{array}{l}\text { Tecnología en Documentación y } \\
\text { Archivística }\end{array}$ & $\begin{array}{l}\text { Tecnólogo en Documentación y } \\
\text { Archivística }\end{array}$ \\
\hline Universidad INPAHU & $\begin{array}{l}\text { Tecnología en Administración de } \\
\text { Sistemas de información y Docu- } \\
\text { mentación. }\end{array}$ & $\begin{array}{l}\text { Tecnólogo en Administración de } \\
\text { Sistemas de Información y Docu- } \\
\text { mentación }\end{array}$ \\
\hline \multicolumn{2}{|c|}{ Denominación de los programas de formación técnica } \\
\hline SENA & \multicolumn{1}{c|}{ Trítulo que Otorga } \\
\hline GADIER & Archivística & Técnico Profesional en Archivística \\
\hline
\end{tabular}

\section{Modalidades y estilos de educación}

Se identificaron dos sectores bien diferenciados en cuanto a los destinatarios de la educación no formal:

- Un primer sector cuyo perfil no es profesional y el cual requiere adquirir conocimientos básicos (fundamentación) que faciliten el desarrollo de su actividad laboral o el cumplimiento de las funciones en un determinado cargo. En este sector se ubica la formación impartida por entidades como el Archivo General de la Nación para desarrollar el Sistema Nacional de Archivos.

- Un segundo sector con un perfil profesional, cuyo interés es actualizar sus conocimientos o adquirir formación complementaria a la recibida en la universidad, bien para mantenerse al día o para aplicar dichos conocimientos en su actividad profesional. Este sector es atendido principalmente por las universidades y por las asociaciones en cumplimiento de sus fines misionales. 
Tabla 10. Tipo y estilo de educación

\begin{tabular}{|c|c|c|c|c|c|c|c|c|c|}
\hline \multirow[b]{3}{*}{$\begin{array}{l}\text { Estilo de } \\
\text { educación }\end{array}$} & \multirow[b]{3}{*}{ 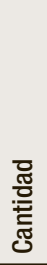 } & \multicolumn{8}{|c|}{ Tipo de educación } \\
\hline & & \multicolumn{2}{|c|}{ Formal } & \multicolumn{6}{|c|}{ No formal } \\
\hline & & 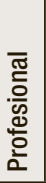 & 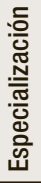 & 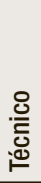 & 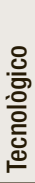 & $\begin{array}{l}\text { के } \\
\text { 壳 }\end{array}$ & 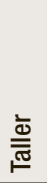 & 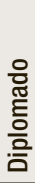 & \\
\hline Presencial & 39 & 1 & 3 & 1 & 2 & 12 & 6 & 3 & 11 \\
\hline Semipresencial & 2 & & 1 & & 1 & & & & \\
\hline Virtual & 3 & 2 & & & & 1 & & & \\
\hline Totales & 44 & 3 & 3 & 1 & 2 & 13 & 6 & 2 & 11 \\
\hline
\end{tabular}

En relación con la educación formal una característica en estos sectores es que en Colombia, a diferencia de algunos países latinoamericanos, se ofrece formación de carácter formal tanto en pregrado como en postgrado en tanto que el perfil de sus programas y el cumplimiento de los estándares de calidad proporcionan un alto nivel de fundamentación teórica y promueven la investigación en el área.

Sólo dos instituciones ofrecen educación virtual, modalidad que facilita la formación para personas cuyas necesidades económicas, de ubicación geográfica o de tiempo, no les permiten optar por programas presenciales.

\section{Jornadas en las que se imparten los diferentes programas académicos}

Debido a la naturaleza del sector, la formación no formal impartida en el sector de archivos se lleva a cabo principalmente durante la jornada diurna, mientras que la formación formal (universitaria) se lleva a cabo en horario nocturno o mixto (diurno y nocturno). De acuerdo con los datos obtenidos los programas identificados según el nivel de formación se imparten en las siguientes jornadas:

Tabla 11. Jornadas de los programas académicos - Archivística

\begin{tabular}{|c|c|c|c|c|c|c|c|c|c|}
\hline \multirow[b]{2}{*}{ Jornada } & \multirow[b]{2}{*}{ 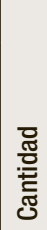 } & \multicolumn{2}{|c|}{ Formal } & \multicolumn{6}{|c|}{ No formal } \\
\hline & & 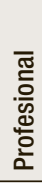 & 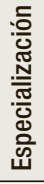 & $\begin{array}{l}\text { 을 } \\
\text { 엍 }\end{array}$ & 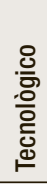 & $\begin{array}{l}\text { क } \\
\text { ज゙ }\end{array}$ & $\frac{\grave{\Xi}}{\stackrel{\bar{\Xi}}{\models}}$ & $\begin{array}{l}\text { 윰 } \\
\text { 흠 } \\
\text { 음 } \\
\text { 은 }\end{array}$ & 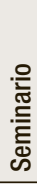 \\
\hline Diurno & 30 & & & 1 & 2 & 11 & 5 & 1 & 11 \\
\hline Nocturno & 10 & & & 1 & 1 & 2 & 1 & 2 & \\
\hline Diurno y Nocturno & 3 & 3 & 3 & & & & & & \\
\hline Total & 43 & 3 & 3 & 1 & 3 & 13 & 6 & 3 & 11 \\
\hline
\end{tabular}


- Los programas con horario de desarrollo nocturno incluyen clases sabatinas.

- Los programas de pregrado y postgrado que se imparten en el sector de archivos captan personas que están vinculadas laboralmente y quienes por tanto no disponen de tiempo para asistir a clases durante su jornada laboral. En cuanto a los postgrados, éstos se realizan en jornadas que incluyen fines de semana (sábados), lo cual les facilita el acceso a quienes tienen vinculaciones laborales y otras responsabilidades que les impiden adelantar estudios en horarios diurno o nocturno exclusivamente.

- En cuanto a la educación no formal, por sus características, ésta se ofrece casi en su totalidad en horario diurno, por cuanto está orientada a la actualización y se dirige a personas que desarrollen alguna actividad en los sectores analizados; además de que se lleva a cabo durante lapsos cortos de tiempo.

La duración de los diferentes programas de tipo no formal se adecua a las necesidades de disponibilidad en tiempo y economía de las personas que optan por adquirir conocimientos a través de este tipo de formación, buscando que los contenidos de estudio que se imparten sean de calidad, cubran los objetivos de formación establecidos y sean aportaciones reales para el desarrollo de las competencias de los trabajadores del sector.

\section{ANÁLISIS DE LA OFERTA DE ACUERDO CON EL NIVEL EDUCATIVO}

Los programas de formación formal que se ofrecen en archivística tienen diferentes denominaciones, aunque se han agregado titulaciones que corresponden a una tendencia que mejore la percepción de la importancia que tienen los archivos dentro de la administración y para la sociedad, y que está asociada al desarrollo tecnológico de las empresas. La oferta formativa responde también a los cambios en las tendencias sobre el manejo de la información y la documentación, los cuales se reflejan en las titulaciones ofrecidas, así como en la orientación y los contenidos de la formación.

No existe un eje temático dominante en la educación no formal en archivos; por el contrario, la oferta identificada es variada tanto en las áreas de formación, como en los tipos de programas, el estilo de educación y la duración. Las instituciones que concentran formación no formal en archivística son el Archivo General de la Nación y el Servicio Nacional de Aprendizaje; en segundo lugar se encuentran las asociaciones profesionales como la Sociedad Colombiana de Archivistas y las asociaciones de egresados de los programas 
de Archivísticas a nivel profesional y en tercer lugar las instituciones privadas relacionadas con el sector.

En el caso de los archivos municipales la formación más habitual es el seminario, seguido de los cursos cortos, en tanto que para los archivos de la administración pública central las modalidades de formación más frecuentes son los diplomados y los postgrados, debido fundamentalmente a que quienes manejan el archivo tienen formación universitaria.

El interés por abrir espacios de formación en los archivos se hace evidente al encontrarse programas en ciudades como Ibagué, Manizales y Armenia, y en general los egresados de los programas de formación universitarios se ubican laboralmente en diferentes ciudades del país. Por su naturaleza virtual, el programa de la Universidad del Quindío cubre todo el territorio nacional y puede captar estudiantes de diferentes regiones del país, ventaja que sin embargo aún no ha sido aprovechada adecuadamente.

\section{Tipo y estilo de educación ofrecida}

En la tabla 12 se resumen los diferentes estilos de educación ofrecidos en el mercado (presencial, semipresencial y virtual) en cada uno de los diferentes niveles de formación (profesional y de postgrado) y los tipos de educación (no formal y formal).

Tabla 12. Tipo y estilo de educación ofrecida

\begin{tabular}{|c|c|c|c|c|c|c|c|c|c|c|}
\hline \multirow[b]{3}{*}{$\begin{array}{l}\text { Estilo de } \\
\text { educación }\end{array}$} & & \multicolumn{9}{|c|}{ Tipo de educación } \\
\hline & & \multicolumn{4}{|c|}{ Formal } & \multicolumn{5}{|c|}{ No formal } \\
\hline & 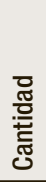 & $\begin{array}{l}\overline{\widetilde{\sigma}} \\
\text { 흠 } \\
\stackrel{0}{0} \\
\text { 은 }\end{array}$ & 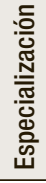 & 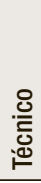 & 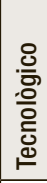 & 总 & $\begin{array}{l}\text { ప } \\
\stackrel{\bar{\sigma}}{\sigma} \\
\end{array}$ & $\begin{array}{l}\text { 율 } \\
\text { हू } \\
\text { 음 }\end{array}$ & 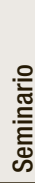 & ? \\
\hline Presencial & 45 & 2 & 3 & 1 & 2 & 13 & 6 & 3 & 11 & 4 \\
\hline Semipresencial & 1 & & 1 & & & & & & & \\
\hline Virtual & 1 & 1 & & & & & & & & \\
\hline Totales & 47 & 3 & 4 & 1 & 2 & 13 & 6 & 3 & 11 & 4 \\
\hline
\end{tabular}

De acuerdo con la información anterior, la educación de tipo no formal prevalece sobre la formal, en términos de la cantidad de programas ofrecidos y de cobertura; y la oferta de captación no formal se concentra en las mismas ciudades en donde se imparte la educación formal.

En relación con la educación formal, una característica en estos sectores es que en Colombia, a diferencia de otros países latinoamericanos, se ofrece formación 
de carácter formal tanto en pregrado como en postgrado (por ahora sólo a nivel de especialización), porque el perfil de sus programas y el cumplimiento de los estándares de calidad para la educación superior proporcionan mayor fundamentación teórica y promueven la investigación en el área.

Si se analiza la oferta de educación no formal en comparación con la formal, se encuentra que los postgrados no reciben exclusivamente profesional con formación universitaria en archivística, sino también a aquellos provenientes de otras disciplinas que requieren formación en el área de archivos, lo cual se debe principalmente a la amplia oferta laboral en el sector.

Los postgrados que se ofrecen actualmente, con excepción de uno, no tienen opciones académicas de estilo semipresencial; a nivel de pregrado, como ya se mencionó, existen dos instituciones que ofrecen educación virtual, modalidad que les facilita la formación a personas cuyas necesidades económicas, de ubicación geográfica o de tiempo no les permiten optar por programas presenciales.

\section{Duración y horarios de la formación en archivos}

\section{Horarios}

Según los datos obtenidos a partir de los 44 programas identificados, 11 se desarrollan en horario nocturno, 30 se ofrecen en jornada diurna, y tres combinan el horario diurno y nocturno; y en cuanto a las especializaciones dos se ofrecen en horario nocturno y los sábados, y una sola en jornada diurna y sábado; y por lo que se refiere a los diplomados, dos se ofrecen en horario nocturno y los días sábados, y sólo uno en horario diurno. Por su parte los programas de educación no formal la mayoría se ofrece en horario diurno (Tabla 13).

Vale la pena aclarar que para los programas con horario de desarrollo nocturno, se incluyen clases durante el día los fines de semana (sábado); y que de los tres programas de pregrado que se ofrecen en dos jornadas (diurna y nocturna), dos de ellos no tienen estudiantes matriculados en la jornada diurna.

En este sentido se puede concluir que:

- Los programas de pregrado y postgrado que se imparten en el sector de archivos captan personas que están vinculadas laboralmente en este sector y quienes por tanto no disponen de tiempo para asistir a clases durante su jornada laboral. En cuanto a los postgrados, los tres se realizan en jornadas que incluyen fines de semana (sábados), 
lo cual facilita su acceso para quienes tienen una vinculación laboral u otras responsabilidades que les impiden adelantar estudios en horarios diurno o nocturno exclusivamente.

- Dadas las características de la educación no formal ésta se ofrece casi en su totalidad en horario diurno, está orientada a la actualización y se dirige a personas que desarrollen alguna actividad en archivos, además de que se dura un tiempo corto.

\section{Duración}

Los tiempos establecidos para el desarrollo en los diferentes programas de tipo no formal varían considerablemente (Tabla 13), porque deben ajustarse a las necesidades de disponibilidad en cuanto al tiempo y la economía de las personas que optan por adquirir conocimientos a través de este tipo de formación.

Tabla 13. Duración de los diferentes programas

\begin{tabular}{|c|c|c|}
\hline \multicolumn{3}{|c|}{ Promedio de duración por nivel de educación } \\
\hline Tipo de educación & Nivel de educación & Promedio \\
\hline \multirow[t]{2}{*}{ Formal } & Profesional & 8-10 semestres \\
\hline & Especialización & 2 semestres \\
\hline \multirow[t]{7}{*}{ No formal } & Técnico & $\begin{array}{l}2 \text { a } 4 \text { semestres (1830 } \\
\text { horas) }\end{array}$ \\
\hline & Tecnológico & 6-7 semestres \\
\hline & Curso & Entre 16 y 40 horas \\
\hline & Taller & $\begin{array}{l}\text { Entres } 16 \text { horas y } 40 \\
\text { horas }\end{array}$ \\
\hline & Diplomado & Entre 90 y 120 horas \\
\hline & Seminario & Entre 16 y 40 horas \\
\hline & Otros & Irregular \\
\hline
\end{tabular}

Asimismo la formación de pregrado y especialización, en cumplimiento con la legislación colombiana que la regula, desarrolla sus contenidos dentro de estándares de tiempo que han sido definidos como adecuados para lograr calidad en el aprendizaje de conocimientos y competencias de sus egresados.

\section{Contenidos y énfasis en la formación}

La formación de pregrado y especialización, en cumpliendo con la legislación que los regula, desarrolla sus contenidos con base en los estándares de tiempo definidos para alcanzar calidad en los conocimientos y competencias que se esperan de un egresado de estos niveles de educación. 
Al revisar los planes de estudio que identifican el contenido académico de cada propuesta de formación universitaria, se observa que para complementar la formación disciplinar, entendida como aquella que apunta al desarrollo de conocimientos específicos del área de la archivística, se han adicionado contenidos de formación orientados hacia las siguientes áreas:

- Administración y gerencia

- Servicios

- Tecnologías de la información

- Procesos técnicos de la función archivística

Según el estudio, las empresas requieren contar con personal que pueda desarrollar actividades técnicas propias de su área de conocimiento, y al mismo tiempo ser capaces de planear y proponer innovaciones en los procesos o en los productos que afectan su quehacer, contribuyendo con su trabajo no sólo en la parte técnica sino generando competitividad para la empresa a la que prestan sus servicios.

Una característica común de los diferentes programas de formación es el campo de acción en el que pueden desempeñarse los egresados, caracterizado por las siguientes áreas:

- Administrativas: planeación, organización, diseño y dirección de archivos y préstamo de asesorías a entidades públicas y privadas en los campos de su formación disciplinar.

- Técnicas: análisis, organización, descripción, recuperación y tratamiento de documentación; diseño, evaluación y prestación de servicios de información; diseño y ejecución de procesos técnicos

- Educativas: apoyar a los usuarios en la búsqueda de información, y capacitarlos en el uso de los servicios y las fuentes de información conservadas en los archivos.

- Investigativas: investigar acerca del uso y consumo de la información; llevar a cabo estudios de comunidad y estudios de usuarios; analizar y evaluar el impacto de los servicios y de las fuentes de información.

De igual forma el análisis ocupacional permite identificar diferencias entre los programas de pregrado, así:

- Sólo dos programas de pregrado ofrecen una formación en el campo archivístico, aunque con diferencias en la cantidad de créditos y el número de asignaturas; otro de ellos ofrece esta formación en modalidad virtual. 
- La formación tecnológica ésta distribuida en Bogotá, Medellín y Manizales; el ultimo de éstos se ofrece en modalidad semipresencial.

- La formación en el campo investigativo y técnico en el campo archivístico es más fuerte en la Universidad de la Salle, tanto en relación con las asignaturas como con el número de créditos.

En cuanto al personal vinculado en archivos municipales, el nivel de capacitación que posee es dispar, y se estima que hay que fortalecer la formación técnica de este personal para que pueda ejercer labores de coordinación de archivos de acuerdo con la categoría de cada municipio sin embargo lo ideal sería que la coordinación del archivo estuviera a cargo de un profesional, aunque se debe tener en cuenta la estructura geopolítica del país y los bajos salarios en la mayoría de los municipios, lo que no favorece el desplazamiento de profesionales hacia las regiones que requieren personal calificado en archivos; por lo tanto una alternativa sería la formación de técnicos o tecnólogos en archivos que puedan atender las necesidades básicas de estos archivos.

También se identificaron funcionarios de archivo que han tomado cursos tanto en archivística como en administración y servicio al cliente, aunque según los resultados de un panel de expertos realizado en el 2006 para obtener información del sector de archivos municipales, un alto número de los archivistas municipales se ha formado de manera autodidacta a partir de las cartillas que ha publicado el AGN, o tomando alguno de los cursos de formación que imparte el propio AGN. Desde el punto de vista de la formación, el nivel básico de formación del personal que trabaja en archivos municipales tiene titulo de bachiller, y en menor proporción algunos cuentan con técnicos del SENA.

Entre las entidades que ofrecen capacitación para este personal se encuentran el AGN, a través del Sistema Nacional de Archivos, los consejos departamentales de archivos y eventualmente otras instituciones como el Banco de la República. Un mecanismo que se está utilizando con buenos resultados son las videoconferencias, que sobre todo han sido organizadas en convenio por el SENA y el AGN. Otros medios de formación utilizados por el personal de estos archivos son cartillas, folletos, libros, o kits mediáticos, compuestos por medios como casetts, videos, $\mathrm{CD}$, disquetts, que envían instituciones como el AGN.

\section{CONCLUSiOnes}

La capacitación profesional en el área de archivos se concentra en la zona andina del país donde operan las universidades que ofrecen estudios en este 
campo; además de ellas las únicas opciones de formación las ofrece el AGN y el SENA, principalmente. Por lo general, la capacitación que ahí se ofrece está orientada a capacitar en las actividades básicas que se desarrollan en los archivos, desde cómo elaborarlo hasta cómo aplicarlo; en muchos casos las personas que acceden a la capitación pueden tener un conocimiento empírico sobre las actividades.

De acuerdo con el estudio las áreas de formación que requieren mayor atención en los archivos del orden municipal del país son las siguientes:

- Planeación

- Historia

- Legislación archivística

- Análisis de información

- Uso y aplicación de tecnologías de la información y la comunicación

- Servicios de información

- Restauración y conservación básicas, que permitan hacer intervenciones en los documentos con prácticas correctas

- Elaboración y aplicación de Tablas de Retención Documental, dando con ello cumplimiento a la legislación

- Descripción de archivos

Existe una oferta de formación muy limitada tanto en relación con la cantidad de programas como con los niveles de educación. Es urgente la creación de nuevos programas de formación formal (conducentes a título) que generen competencias relacionadas con la capacidad de gestión, desarrollen habilidades para la ejecución de labores rutinarias y les permitan a los alumnos autoevaluar su desarrollo, además de cumplir con la legislación en la materia; y otro tema necesario es la capacitación para realizar diagnósticos, y diseñar y ejecutar planes.

En el sector municipal sigue siendo frecuente encontrar que los funcionarios públicos no reconocen el archivo como un bien de la organización y por lo tanto los archivistas municipales no apoyan su administración municipal, lo que impide el desarrollo de una adecuada gestión de los archivos. Se menosprecia la labor de archivo y éste acepta los elementos que no utilizan las demás áreas de la institución, porque se considera que "el archivo" no puede obtener mejores recursos. Resulta pues necesario fortalecer la motivación y la convicción de que el archivo es una herramienta de apoyo a la gestión de la administración municipal, y también resulta necesario que el Departamento Administrativo de la Función Pública, conjuntamente con el AGN y las asociaciones profesionales del sector, definan el perfil del archivista municipal 
de acuerdo con las características de los cargos que ofrecen la administración pública y el nivel del municipio.

Si bien se evidencia un aumento en el número de personas que trabajan en archivos y han recibido capacitación en estos temas, al evaluar la gestión realizada en éstos se establece que esta formación ha sido tomada en algunos casos con el propósito de acumular créditos académicos que les permitan ascender en el municipio, sin que esto implique compromiso en la aplicación de los conocimientos recibidos.

En la mayoría de casos las personas que aceptan la capacitación no continúan en los siguientes niveles o programas complementarios porque cambian de cargo, y por tanto de funciones, dentro de la misma administración municipal.

Como conclusión general, la educación superior universitaria en este sector busca mejorar los conocimientos y habilidades de los profesionales proveyendo conocimientos que les permitan resolver problemas, mediante la evaluación de situaciones reales y la toma de decisiones oportunas frente a cambios políticos, económicos, sociales y tecnológicos. Al revisar los planes de estudio que identifican el contenido académico de cada propuesta de formación universitaria, se observa que para complementar la formación disciplinar, entendida como aquella que apunta al desarrollo de conocimientos específicos en archivística, se han adicionado contenidos de formación orientados hacia áreas como administración y gerencia, servicios, tecnologías de la información y procesos de la función archivística.

En relación con los perfiles profesionales, los diferentes programas de formación tienen una visión común del campo disciplinar, y en general se puede decir que los contenidos que se imparten tienen un perfil muy influenciado por las políticas nacionales que en materia archivística, promulga el Archivo General de la Nación, y los directrices de organismos como el Consejo Internacional de Archivos y la UNESCO.

\section{BIBLIOGRAFIA}

Alberch I Fugueras, Ramón, Los archivos municipales en el contexto internacional, en Seminario del Sistema Nacional de Archivos (7, 25-27. 1998, Santa fe de Bogotá), Memorias, Archivos Municipales / Archivo General de la Nación de Colombia, División de programas especiales, Santa fe de Bogotá, Archivo General de la Nación, 1999.

Anónimo, Principio del análisis técnico: Los principios de las tendencias, [En línea], disponible en: <http://elaccionista.com/tecnico/ TENDENCIAS.htm> 
Asociación Colombiana De Bibliotecólogos y Documentalistas - ASCOLBI, Mesa sectorial de competencias laborales en bibliotecología, [en línea], disponible en: <http: www.ascolbi.org/documentos/noticia_mesa_de_trabajo.htm.>

Archivo General de la Nación, Relación de temas de los seminarios del Sistema Nacional de Archivos (1992 -2005) / XIV Seminario, Los archivos y la gestión del conocimiento: valoración documental (Nov. 2004), Bogotá, 21 de Septiembre 2005.

Camacho, Angela Marcela y Caro Sandoval, Marcela, Estudio de caracterización ocupacional en archivos de la administración pública en Colombia, Bogotá, Universidad de la Salle, 2006, [Tesis de Grado], 141p.

Cinterfor, Organización internacional del trabajo. 40 Preguntas sobre competencia laboral: 35 ¿Qué es un sistema nacional de formación? [en línea], Montevideo: Cinterfor, 2001. 4 p, www.cinterfor.org.uy

Colombia, Archivo General de la Nación, AGN. Censo guía y estadística de los archivos colombianos, Bogotá, El Archivo, 1991.

Noticias del Archivo General de la Nación: Eventos de capacitación archivística 2005, Cursos, talleres, seminarios, foros, [en línea], disponible en: <http://www.archivogeneral.gov.co/ version $2 />$

, Seminario del Sistema Nacional de Archivos. Memorias, (Noviembre 4,5 y 6 de 1992: Santafe de Bogotá), Santafe de Bogotá, El Archivo, 1993.

Seminario sobre formación y capacitación archivística, Memorias, (Julio 18 y 19 de 1995: Santafé de Bogotá), Santafé de Bogotá, El Archivo, 1996.

Sistema Nacional de Archivos: Archivos Municipales. Memorias, Santafé de Bogotá, Archivo General de la Nación de Colombia, Agosto 25-27 de 1998.

, Sistema Nacional de Archivos: Balance y Perspectivas. Memorias, Bogotá, Archivo General de la Nación de Colombia, octubre 27, 28 y 29 de 1999.

Sistema Nacional de Archivos: Balance y Perspectivas. Memorias, Bogotá, AGN, 2005.

Colombia, Ministerio de Educación Nacional, Formar para el trabajo, construir el país, Bogotá, "El ministerio", en Educación superior, Boletín informativo, Núm. 3, octubre - diciembre, 2004, 18 p.

Ley 30: organiza el servicio público de la Educación Superior, Bogotá, Ministerio de Educación Nacional, 1992.

, Ley 115: Ley general de educación, Bogotá, Ministerio de Educación Nacional, 1994.

, Ley 594: Ley general de archivos, Bogotá, Archivo General de la Nación, 2000. 
Ley 909 de 2004: Expiden normas que regulan el empleo público, la carrera administrativa, gerencia pública. Titulo V, El ingreso y el ascenso a los empleos de carrera, Capítulo I, Art. 27, [en línea], disponible en: <http://www.udenar.edu.co/derechopublico/Ley909_2004(C_A).htm>

COLSUBSIDIO, Auxiliar de oficina con énfasis en Documentación y Archivo, [en línea], disponible en: <http://www.comfenalcocundinamarca.com/f-educacion.htm

COMfama: Escuela de Servicios Administrativos y de Mercadeo. Técnico en Administración de Sistemas de Información, [en línea], disponible en: <http:/www.comfama.com/contenidos/servicios/ Educaci\%F3n/Escuela\%20de\%20Servicios\%20Admitivos\%20 y\%20de\%20Mercadeo/Programas\%20T\%E9cnicos/ProgramasTecnicosOcupacionales.asp $>$.

Técnicas de oficina: Organización de archivos, [en línea], Medellín, COMFAMA, 2005, disponible en: http://www.comfama. com/t_matri/por_categoria.asp?cod_cate $=114 \&$ estado $=$ inicio.

COMFAMILIAR - Atlántico, Diplomado en: Gerencia Documental orientado a la gestión de archivos históricos y empresariales, [en línea], disponible en: http://www.comfamiliar.com.co/Modulos/ Educacion/educacion_corpoeducativa.asp.

COMFAMILIAR - Quindío, Administración de archivo y manejo de documentos, [en línea], disponible en: <http://www.comfamiliarquindio.com.co/capacitacion.htm>

Consejo Internacional de Archivos, [en línea], disponible en: <http:// www.ica.org/static.php?ptextid $=$ sommaire \&plangue $=$ es $>$.

Corporación Educativa Mayor del Desarrollo Simón Bolivar, Noticias Asouniesca [en línea], disponible en: <http://www.unisimonbolivar.edu.co/modulos/servicios/submenu.php? codigo $=2 \& o p=1 \&$ portal $=$ ser \&nivel $=2 \&$ submenu $=8 \&$ tipo $=18>$.

INPAHU, Fundación Escuela Superior Profesional, Tecnólogo en administración de Sistemas de Información y Documentación, [en línea], disponible en: http://www.inpahu.edu.co/aspirantes/Programas\%20Acade/admon-documenta.htm.

Pontificia Universidad Javeriana, Facultad de comunicación y lenguaje. Ciencia de la información, Especialización en archivística, [en línea], disponibilidad en: <http://www.javeriana.edu.co/puj/posgrados.htm.>, consulta (Diciembre 5, 2005).

Diplomado: Gestión de información documental - una perspectiva para la administración de información y conocimiento, Bogotá, La Universidad, 2005, 2 p.

, Especialización en redes de información documental, [en línea], disponible en: <http://www.javeriana.edu.co/puj/posgrados.htm>

Rossini, Daniel, Los archivos y las nuevas tecnologías de la información, La Paz: Congreso Internacional de Bibliotecología, Documentación y Archivística (CIBDA), 2003. 
Servicio Nacional de Aprendizaje - SENA, Metodología para la elaboración de normas de competencia laboral, [término de búsqueda: estudios de caracterización], [en línea]. [pdf], Bogotá, SENA, 2003, [consulta: 17 jul., 2006], 97 p., disponible en: http://www. tlc.gov.co/VBeContent/Documentos/contratacion/2004/ contrataciondirecta/27_Dise\%C3\%B1ocurricular/Anexo_6_ MetodologiaNCL2003.pdf.

, Oficina Virtual, Fundamentos de administración documental, [en línea], disponible en: <http://oficina.senavirtual.edu. co/main_oferta.html>.

, Sedes en las regionales. Técnico en archivística: Técnico, [en línea], disponible en: $<$ http://200.21.18.179/letras.aspx?Regional=5

Sociedad Colombiana de Archivistas - SCA, Cursos de capacitación 2005, [en línea]. disponible en: <http://www.sociedadcolombianadearchivistas.org/capacitacion.htm $>$.

Presentación, [en línea], disponible en <http://www.sociedadcolombianadearchivistas.org/presentacion.htm $>$. , Programa de capacitación 2003 -2004, Bogotá, SCA, 2005, $4 \mathrm{p}$.

Universidad del Quíndio, Programa académico: Ciencia de la Información y la Documentación, Bibliotecología y Archivística, [en línea], disponible en: <http://www.uniquindio.edu.co/uniquindio/index.htm $>$.

Universidad de la Salle, División de Formación Avanzada. Especialización en Sistemas de Información y Gerencia de Documentos, [en línea], disponible en: <http://www.lasalle.edu.co/postgrado/ postgrados/esp_sistemas.htm $>$.

, Facultad de sistemas de información y documentación, Modernización curricular año 2002, Bogotá, La Facultad, 2002, $133 \mathrm{p}$.

Sistemas de Información y Documentación, Egresados, [en línea], disponible en: <http://www.lasalle.edu.co/pregrado/ preg_sist_inf_docum/egresados.htm $>$.

Universidad Pedagógica y Tecnológica de Colombia, Centro de Investigaciones y Extensión de la Facultad de Ciencias de la Educación (CIEFED), [en línea], disponible en: <http://www.uptc.edu.co/ universidad/en/index.html>

Yate Parra, Paola Fernanda y Castaño Zuluaga, Diana Marcela, Estudio Exploratorio de Casos de Outsourcing de Servicios de Archivo Aplicado a Empresas Públicas y Privadas en Bogotá, Bogotá, 2004, 86 p., Trabajo de grado (Profesional en Ciencia de la Información - Bibliotecología), Pontificia Universidad Javeriana, Colombia, Facultad de comunicación y lenguaje, Carrera de ciencia de la información.

Zapata García, León Jaime, La Asociación Colombiana de Bibliotecarios: apuntes y documentos para su historia, [Tesis], Bogotá, Universidad Social Católica de la Salle, 1977. 


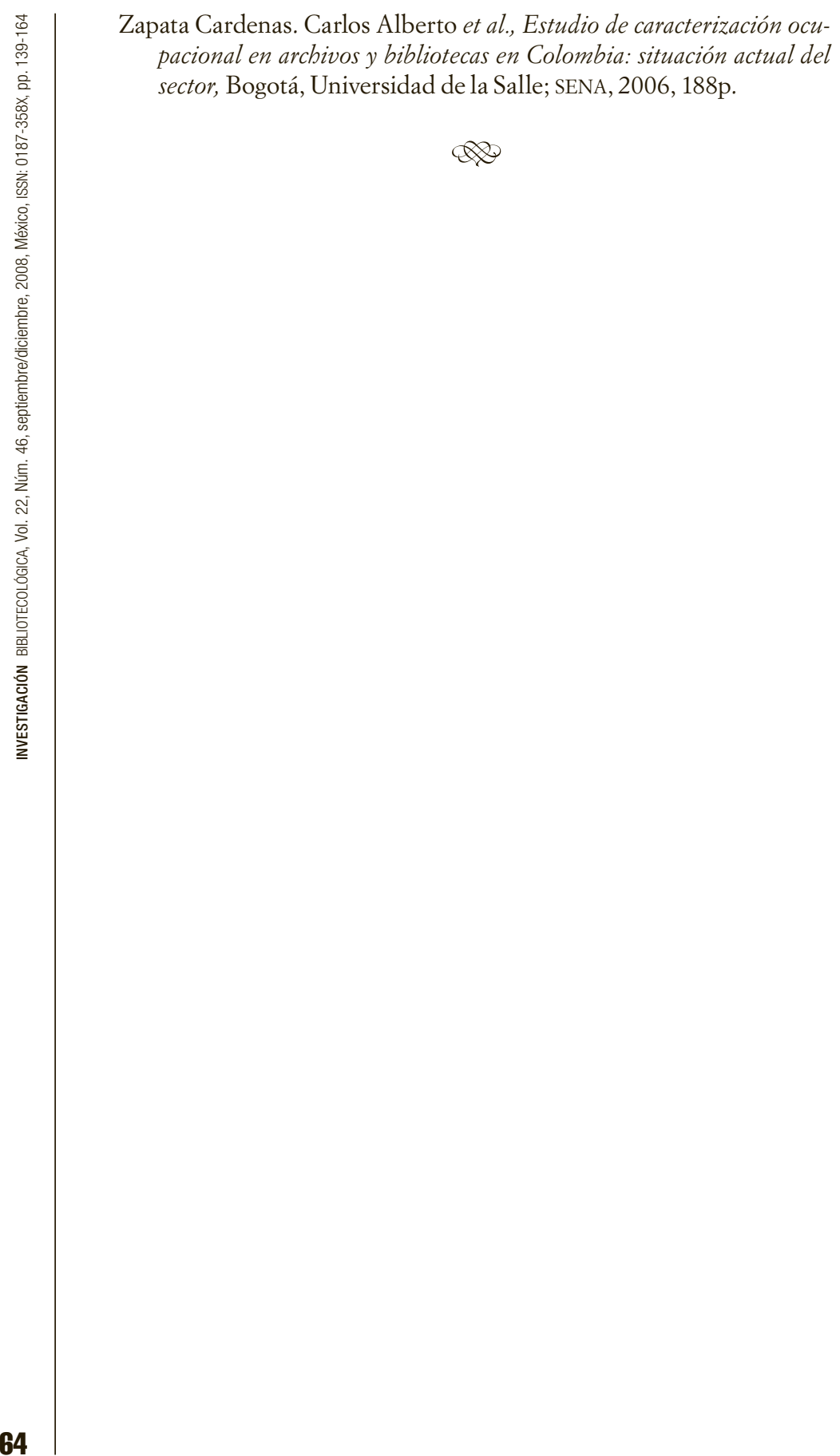

